\title{
Existence of a Nontrivial Solution for a Class of Superquadratic Elliptic Problems*
}

\author{
Xiuming Mo" ${ }^{1}$, Ping $\mathrm{Jing}^{2}$, Yan Zhao ${ }^{3}$, Anmin Mao" ${ }^{4 \#}$ \\ ${ }^{1}$ Department of Biotechnology, Beijing City University, Beijing, China \\ ${ }^{2}$ School of Mathematics and Statistics, Central South University, Changsha, China \\ ${ }^{3}$ Employee's College of Dongcheng in Beijing, Beijing, China \\ ${ }^{4}$ School of Mathematical Sciences, Qufu Normal University, Qufu, China \\ Email: "maoam@163.com
}

Received May 17, 2012; revised June 20, 2012; accepted June 28, 2012

\section{ABSTRACT}

We consider the existence of a nontrivial solution for the Dirichlet boundary value problem

$$
\begin{cases}-\Delta u+a(x) u=g(x, u), & \text { in } \Omega, \\ u=0, & \text { on } \partial \Omega .\end{cases}
$$

We prove an abstract result on the existence of a critical point for the functional $f$ on a Hilbert space via the local linking theorem. Different from the works in the literature, the new theorem is constructed under the $(C)^{*}$ condition instead of $(P S)^{*}$ condition.

Keywords: Elliptic Problems; Local Linking Theorem; $(C)^{*}$ Condition; Superquadratic

\section{Introduction and Main Results}

Consider the Dirichlet boundary value problem

$$
\begin{cases}-\Delta u+a(x) u=g(x, u), & \text { in } \Omega, \\ u=0, & \text { on } \partial \Omega,\end{cases}
$$

where $a(x) \in L^{p}(\Omega), p>N / 2, g \in C(\bar{\Omega} \times R, R)$ and $\Omega \subset R^{N}(N \geq 3)$ is a bounded domain whose boundary is a smooth manifold.

We assume that $G$, where $G(x, u)=\int_{0}^{u} g(x, s) \mathrm{d} s$. In [1], Li and Willem established the existence of a nontrivial solution for problem (1) under the following well-known Ambrosetti-Rabinowitz superlinearity condition: there exists $\mu>2$ and $L>0$ such that

$$
0<\mu G(x, u)<u g(x, u)
$$

for all $|u| \geq L$ and $x \in \Omega$, which has been used extensively in the literature; see [1-4] and the references therein. It is easy to see that condition $(A R)$ does not include some superquadratic nonlinearity like

$$
G(x, u)=|u|^{2}\left(\ln \left(\frac{1}{3}|u|^{4}-|u|^{2}+1\right)\right)^{3}
$$

In [5], Qin Jiang and Chunlei Tang completed the Theorem 4 in [1], and obtained the existence of a nontrivial solution for problem (1) under a new superquadratic condition which covered the case of (G0). The conditions are as follows:

(G1) $G(x, u) /|u|^{2} \rightarrow+\infty$, as $|u| \rightarrow+\infty$ uniformly on $\Omega$ $\Omega$,

(G2) $G(x, u) /|u|^{2} \rightarrow 0$, as $|u| \rightarrow 0$ uniformly on

(G3) There are constants $1<\lambda<\frac{N+2}{N-2}$ and $a_{1}>0$ such that

$$
|g(x, u)| \leq a_{1}\left(|u|^{\lambda}+1\right)
$$

for all $(x, u) \in \Omega \times R$,

(G4) There are constants $\beta>\frac{2 N}{N+2} \lambda, a_{2}>0$ and $L>0$ such that

$$
u g(x, u)-2 G(x, u) \geq a_{2}|u|^{\beta}
$$

for all $|u| \geq L$ and $x \in \Omega$,

If 0 is an eigenvalue of $-\Delta+a$ (with Dirichlet boundary condition) assume also the condition that:

(G5) There exists $\delta>0$ such that: 
1) $G(x, u) \geq 0$, for all $|u| \leq \delta, \quad x \in \Omega$; or

2) $G(x, u) \leq 0$, for all $|u| \leq \delta, x \in \Omega$.

Note that (G4) is also (AR) type condition.

The aim of this paper is to consider the nontrivial solution of problem (1) in a more general sense. Without the Ambrosetti-Rabinowitz superlinearity condition $(A R)$ or (G4), the superlinear problems become more complicated. We do not know in our situations whether the $(P S)$ or $(P S)^{*}$ sequence are bounded. However, we can check that any Cerami (or $\left.(C)^{*}\right)$ sequence is bounded. The definition of $(P S)^{*}$ (or $(C)^{*}$ ) sequence can be found in [6].

We will obtain the same conclusion under the $(C)^{*}$ condition instead of $(P S)^{*}$ condition. So we only need the following conditions instead of (G3) (G4):

(G3') Let $\tilde{G}=\frac{1}{2} g(x, u) u-G(x, u)$ satisfying

1) $\tilde{G} \geq a_{3}|u|^{\beta}$ if $|u| \geq R$,

2) $|g(x, u)|^{\sigma} /|u|^{\sigma} \leq a_{4} \tilde{G}(x, u)$ if $|u| \geq R$, where

$a_{3}, a_{4}>0, \sigma>\frac{N}{2}+1, q=\frac{\sigma+1}{\sigma-1}, \beta>q+1$.

It is easy to see that function

$$
G(x, u)=\frac{1}{2}|u|^{\frac{8}{3}}+|u|^{2} \ln \left(1+|u|^{2}\right)
$$

satisfies conditions of (G1) (G2) (G5) and (G3').

Our main result is the following theorem:

Theorem 1.1. Suppose that $G$ satisfies (G1) (G2) (G5) and (G3'). If 0 is an eigenvalue of $-\Delta+a$ (with Dirichlet boundary condition). Then problem (1) has at least one nontrivial solution.

Remark 1. There are many functions which are superlinear but it is not necessary to satisfy AmbrosettiRabinowitz condition. For example,

$$
\begin{aligned}
& f(x, u)=\theta|u|^{\theta-2} u+(\theta-1)|u|^{\theta-3} u \sin ^{2} u+|u|^{\theta-1} \sin 2 u, \\
& u \in R \backslash\{0\}
\end{aligned}
$$

where $\mu>2$. Then it is easy to check that $(A R)$ does not hold. On the other hand, in order to verify $(A R)$, it usually is an annoying task to compute the primitive function of $f$ and sometimes it is almost impossible. For example,

$$
f(x, u)=|u| u\left(1+e^{(1+\mid \sin u)^{\alpha}}+|\cos u|^{\alpha}\right), \quad u \in R
$$

where $\alpha>0$.

Remark 2. Our condition is much weaker than $(A R)$ type condition (cf. [6]).

\section{Proof of Theorem}

Define a functional $f$ in the space $E=H_{0}^{1}(\Omega)$ by

$$
f(u)=\frac{1}{2}\left(\left\|u^{+}\right\|^{2}-\left\|u^{-}\right\|^{2}\right)-\int_{\Omega} G(x, u) \mathrm{d} x,
$$

where $u^{-} \in E^{-}, u^{+} \in E^{+}, E^{+}\left(E^{-}\right)$is the space spanned by the eigenvectors corresponding to negative (positive) eigenvalue of $-\Delta+a$.

In this paper, we shall use the following local linking theorem (Lemma 2.1) to prove our Theorem. Let $X$ be a real Banach space with $X=X^{1} \oplus X^{2}$ and $X_{0}^{j} \subset X_{1}^{j} \subset \cdots \subset X^{j}$ such that $X^{j}=\bigcup_{n \in N} X_{n}^{j}$, $j=1,2$. For every multi-index $\alpha=\left(\alpha_{1}, \alpha_{2}\right) \in N^{2}$, let $X_{\alpha}=X_{\alpha_{1}}^{1} \oplus X_{\alpha_{2}}^{2}$. We know that $\alpha \leq \beta \Leftrightarrow \alpha_{1} \leq \beta_{1}$, $\alpha_{2} \leq \beta_{2}$. A sequence $\left(\alpha_{n}\right) \subset N^{2}$ is admissible if for every $\alpha \in N^{2}$ there is $m \in N$ such that

$n \geq m \Rightarrow \alpha_{n} \geq \alpha$. We say $f \in C^{1}(X, R)$ satisfies the $(C)^{*}$ condition if every sequence $\left(u_{\alpha_{n}}\right)$ such that $\left(\alpha_{n}\right)$ is admissible and satisfies

$$
u_{\alpha_{n}} \in X_{\alpha_{n}}, \operatorname{supf}\left(u_{\alpha_{n}}\right)<\infty,\left(1+\left\|u_{\alpha_{n}}\right\|\right) f^{\prime}\left(u_{\alpha_{n}}\right) \rightarrow 0
$$

contains a subsequence which converges to a critical point of $f$.

Lemma 2.1. ([6]) Suppose that $f \in C^{1}(X, R)$ satisfies the following assumptions:

$\left(f_{1}\right) \quad f$ has a local linking at 0 ,

$\left(f_{2}\right) f$ satisfies $(C)^{*}$ condition,

$\left(f_{3}\right) f$ maps bounded sets into bounded sets,

$\left(f_{4}\right)$ For every $m \in N, f(u) \rightarrow-\infty$ as $\|u\| \rightarrow \infty$, on $u \in X_{m}^{1} \oplus X^{2}$.

Then $f$ has at least two critical points.

Proof of Theorem 1. We shall apply Lemma 2.1 to the functional $f$ associated with (1), we consider the case where 0 is an eigenvalue of $-\Delta+a$ and

$$
G(x, u) \leq 0 \text { for }|u| \leq \delta .
$$

The other case are similar.

1) $f \in C^{1}(X, R)$ and $f$ maps bounded sets into bounded sets.

Let $X^{2}=X^{-}, \quad X^{1}=X^{+} \oplus X^{0}$. Choose Hilbertian basis $\left(e_{n}\right)_{n \geq 1}$ for $X^{1}$ and $\left(e_{n}\right)_{n \leq-1}$ for $X^{2}$, define

$$
\begin{gathered}
X_{n}^{1}:=\operatorname{span}\left\{e_{1}, \cdots, e_{n+1}\right\}, n \in N ; \\
X_{n}^{2}:=\operatorname{span}\left\{e_{-1}, \ldots, e_{-n-1}\right\}, n \in N .
\end{gathered}
$$

Assumption (G3') implies there are constants $C_{1}, C_{2}>0$ such that

$$
|g(x, u)| \leq C_{1}+C_{2}|u|^{q},
$$

so

$$
\begin{aligned}
|G(x, u)| & =\left|\int_{0}^{1} g(x, s u) u \mathrm{~d} s\right| \\
& \leq \int_{0}^{1}\left(C_{1}|u|+C_{2}|s|^{q}|u|^{q+1}\right) \mathrm{d} s \\
& \leq C_{3}+C_{4}|u|^{q+1},
\end{aligned}
$$


where $q=\frac{\sigma+1}{\sigma-1}>1$.

Hence $f \in C^{1}(X, R)$ and maps bounded sets into bounded sets.

In fact,

$$
\begin{aligned}
|f(u)| & =\left|\frac{1}{2}\left\|u^{+}\right\|^{2}-\frac{1}{2}\left\|u^{-}\right\|^{2}-\int_{\Omega} G(x, u) \mathrm{d} x\right| \\
& \leq \frac{1}{2}\|u\|^{2}+\int_{\Omega}\left(C_{3}+C_{4}|u|^{q+1}\right) \\
& \leq \frac{1}{2}\|u\|^{2}+|\Omega| C_{3}+C_{5}\|u\|,
\end{aligned}
$$

so $\left(f_{3}\right)$ holds.

2) $f$ has a local linking at 0 .

It follows from (G2) and (G3) that, for any $\varepsilon>0$, there exists $C_{\varepsilon}>0$, such that

$$
|G(x, u)| \leq \varepsilon|u|^{2}+C_{\varepsilon}|u|^{q+1},
$$

we obtain, on $X^{2}$, for some $c>0$,

$$
\begin{aligned}
f(u) & \leq-\frac{1}{2}\|u\|^{2}+\varepsilon \int_{\Omega}|u|^{2}+C_{\varepsilon} \int_{\Omega}|u|^{q+1} \\
& \leq-\frac{1}{2}\|u\|^{2}+\varepsilon C_{6}\|u\|^{2}+C_{7}\|u\|^{q+1} \\
& =\left(-\frac{1}{2}+\varepsilon C_{6}+C_{7}\|u\|^{q-1}\right)\|u\|^{2},
\end{aligned}
$$

choosing $r_{1}=\frac{1}{\sqrt[q-1]{4 C_{7}}}$, then $f(u) \leq 0, u \in X^{2}, \quad\|u\| \leq r_{1}$.

Decompose $X^{1}$ into $V+W$ when

$V=\operatorname{ker}(-\Delta+a), W=\left(X^{2}+V\right)^{\perp}$. Also set $u=v+w, u \in X^{1}, v \in V, w \in W$. Since $V$ is a finitedimensional space, there exists $C>0$, such that

$$
\|v\|_{\infty} \leq C\|v\|, \forall v \in V .
$$

First we set $\|u\| \leq \delta / 2 C$ and

$$
\Omega_{1}=\{x \in \Omega|| w(x) \mid \leq \delta / 2\}, \Omega_{2}=\Omega \backslash \Omega_{1} .
$$

On $\Omega_{1}$, we have, by (5)

$$
|u(x)| \leq|v(x)|+|w(x)| \leq\|v\|_{\infty}+\delta / 2 \leq \delta,
$$

hence, by (2)

$$
\int_{\Omega_{1}} G(x, u) \mathrm{d} x \leq 0 .
$$

On $\Omega_{2}$, we have also by (5)

$$
|u(x)| \leq|v(x)|+|w(x)| \leq 2|w(x)|,
$$

hence, by (4)

$$
\begin{aligned}
G(x, u) & \leq \varepsilon u^{2}+C_{\varepsilon}|u|^{q+1} \\
& \leq 4 \varepsilon w^{2}+2^{q+1} C_{\varepsilon}|w|^{q+1},
\end{aligned}
$$

and for some $c>0$

$$
\int_{\Omega_{2}} G(x, u) \mathrm{d} x \leq \int_{\Omega_{2}}\left(4 \varepsilon w^{2}+c|w|^{q+1}\right) .
$$

Therefore we deduce that

$$
\begin{aligned}
f(u) & \geq \frac{1}{2}\|w\|^{2}-\int_{\Omega_{2}}\left(4 \varepsilon w^{2}-c|w|^{q+1}\right)-\int_{\Omega_{1}} G(x, u) \\
& \geq \frac{1}{2}\|w\|^{2}-\varepsilon C_{8}\|w\|^{2}-C_{9}\|w\|^{q+1} \\
& =\left(\frac{1}{2}-\varepsilon C_{8}-C_{9}\|w\|^{q-1}\right)\|w\|^{2},
\end{aligned}
$$

choosing $r_{2}=\frac{1}{\sqrt[q-1]{4 C_{9}}}$, then $f(u) \geq 0, u \in X^{1},\|u\| \leq r_{2}$,

Let $r=\min \left\{\frac{\delta}{2 C}, r_{1}, r_{2}\right\}$, then $\left(f_{1}\right)$ holds.

3) $f$ satisfies $(C)^{*}$ condition.

Consider a sequence $\left(u_{\alpha_{n}}\right)$ such that $\left(\alpha_{n}\right)$ is admissible and

$$
\begin{aligned}
& u_{\alpha_{n}} \in X_{\alpha_{n}}, c:=\sup _{n} f\left(u_{\alpha_{n}}\right)<\infty, \\
& \left(1+\left\|u_{\alpha_{n}}\right\|\right) f^{\prime}\left(u_{\alpha_{n}}\right) \rightarrow 0 .
\end{aligned}
$$

I) $\left(u_{\alpha_{n}}\right)$ is bounded.

For $n$ large, from assumption (G3'), with $u_{n}=u_{\alpha_{n}}$, for some $c>0, m>0$,

$$
\begin{aligned}
c & \geq f\left(u_{n}\right)-\frac{1}{2} f^{\prime}\left(u_{n}\right) u_{n}=\int_{\Omega} \tilde{G}\left(x, u_{n}\right) \\
& \geq \int_{\Omega_{R}} a_{3} u_{n}^{\beta}+\int_{\Omega_{R}^{c}} \tilde{G}\left(x, u_{n}\right) \\
& \geq \int_{\Omega_{R}} a_{3} u_{n}^{2}+\int_{\Omega_{R}^{c}} \tilde{G}\left(x, u_{n}\right) \geq \int_{\Omega_{R}} a_{3} u_{n}^{2}+m,
\end{aligned}
$$

where $\Omega_{R}=\{x \in \Omega|| u(x) \mid>R\}, \Omega_{R}^{c}=\Omega \backslash \Omega_{R}$.

So

$$
\int_{\Omega} u_{n}^{2}=\int_{\Omega_{R}} u_{n}^{2}+\int_{\Omega_{R}^{c}} u_{n}^{2} \leq c .
$$

Arguing indirectly, assume $\left\|u_{n}\right\| \rightarrow \infty$. Set $v_{n}=u_{n} /\left\|u_{n}\right\|$, Then $\left\|v_{n}\right\|=1$ and $\left|v_{n}\right|_{s} \leq C_{s}$ for all $s \in[1, \infty)$. In addition, using (6)

$$
\int_{\Omega} v_{n}^{2}=\frac{1}{\left\|u_{n}\right\|^{2}} \int_{\Omega} u_{n}^{2} \leq \frac{c}{\left\|u_{n}\right\|^{2}} \rightarrow 0,
$$

hence by Interpolation inequality for $L^{p}$ norms, for $s \in[1, \infty)$

$$
\int_{\Omega}\left|v_{n}\right|^{s} \leq\left(\int_{\Omega}\left|v_{n}\right|^{t}\right)^{(1-\theta) s}\left(\int_{\Omega}\left|v_{n}\right|^{2}\right)^{s \theta} \rightarrow 0,
$$

where $\frac{1}{s}=\frac{\theta}{2}+\frac{1-\theta}{t}, 1 \leq t \leq s \leq 2$ or $2 \leq s \leq t \leq \infty$.

Since $\operatorname{dimker}(-\Delta+a)<\infty$, 


$$
\begin{aligned}
& f^{\prime}\left(u_{n}\right)\left(u_{n}^{+}-u_{n}^{-}\right) \\
& =\left\|u_{n}^{+}+u_{n}^{-}\right\|^{2}-\int_{\Omega} g\left(x, u_{n}\right)\left(u_{n}^{+}-u_{n}^{-}\right) \mathrm{d} x \\
& =\left\|u_{n}\right\|^{2}-\left\|u_{n}^{0}\right\|^{2}-\int_{\Omega} g\left(x, u_{n}\right)\left(u_{n}^{+}-u_{n}^{-}\right) \mathrm{d} x \\
& =\left\|u_{n}\right\|^{2}\left(1-\int_{\Omega} \frac{g\left(x, u_{n}\right)\left(u_{n}^{+}-u_{n}^{-}\right)}{\left\|u_{n}\right\|^{2}} \mathrm{~d} x\right)-\left\|u_{n}^{0}\right\|^{2} \\
& \geq\left\|u_{n}\right\|^{2}\left(1-\int_{\Omega} \frac{g\left(x, u_{n}\right)\left(u_{n}^{+}-u_{n}^{-}\right)}{\left\|u_{n}\right\|^{2}} \mathrm{~d} x\right)-c\left|u_{n}^{0}\right|_{2}^{2},
\end{aligned}
$$

So

$$
1-\int_{\Omega} \frac{g\left(x, u_{n}\right)\left(u_{n}^{+}-u_{n}^{-}\right)}{\left\|u_{n}\right\|^{2}} \mathrm{~d} x=o(1) .
$$

From (7) for some $c>0$

$$
\begin{aligned}
& \left|\int_{\Omega} \frac{g\left(x, u_{n}\right)\left(u_{n}^{+}-u_{n}^{-}\right)}{\left|u_{n}\right|^{2}}\right| \leq 2 \int_{\Omega} \frac{g\left(x, u_{n}\right)}{\left|u_{n}\right|}\left|v_{n}\right|^{2} \\
& \leq c\left(\int_{\Omega}\left(\frac{g\left(x, u_{n}\right)}{\left|u_{n}\right|}\right)^{\sigma}\right)^{\frac{1}{\sigma}}\left(\int_{\Omega}\left|v_{n}\right|^{2 \sigma}\right)^{\frac{1}{\sigma^{\prime}}} \\
& \leq c\left(\int_{\Omega}\left|v_{n}\right|^{2 \sigma^{\prime}}\right)^{\frac{1}{\sigma^{\prime}}} \rightarrow 0,
\end{aligned}
$$

as $n \rightarrow \infty$, therefore, $1=o(1)$, a contradiction. Hence $(C)^{*}$ sequence is bounded.

II) From (I) we see that $\left(u_{n}\right)$ is bounded in $X$, going if necessary to a subsequence, we can assume that $u_{n} \rightarrow u$ in $X$. Since dimker $(-\Delta+a)<\infty, u_{n}^{0} \rightarrow u^{0}$ in $X$.

$$
\begin{aligned}
\left\|u_{n}^{+}-u^{+}\right\|^{2}= & \left(f^{\prime}\left(u_{n}\right)-f^{\prime}(u)\right)\left(u_{n}^{+}-u^{+}\right) \\
& +\int_{\Omega}\left(g\left(x, u_{n}\right)-g(x, u)\right)\left(u_{n}^{+}-u^{+}\right)
\end{aligned}
$$

which implies that $u_{n}^{+} \rightarrow u^{+}$in $X$. Similarly, $u_{n}^{-} \rightarrow u^{-}$in $X$. It follows then that $u_{n} \rightarrow u$ in $X$ and $f^{\prime}(u)=0$.

4) Finally, we claim that, for every $m \in N$,

$$
f(u) \rightarrow \infty,\|u\| \rightarrow \infty, u \in X_{m}^{1} \oplus X^{2} .
$$

Indeed, by (G1) we have, there exists $M>0, R>0$ such that

$$
G(x, u) \geq M|u|^{2},|u| \geq R,
$$

so on $X_{m}^{1} \oplus X^{2}$,

$$
\begin{aligned}
f(u) & =\frac{1}{2}\left\|u^{+}\right\|^{2}-\frac{1}{2}\left\|u^{-}\right\|^{2}-\int_{\Omega} G(x, u) \\
& \leq \frac{1}{2}\left\|u^{+}\right\|^{2}-\frac{1}{2}\left\|u^{-}\right\|^{2}-M\left|u^{+}\right|_{2}^{2}-M\left|u^{0}\right|_{2}^{2} \\
& \leq \frac{1}{2}\left\|u^{+}\right\|^{2}-\frac{1}{2}\left\|u^{-}\right\|^{2}-M C\left\|u^{+}\right\|^{2}-M C\left\|u^{0}\right\|^{2} \rightarrow-\infty, \\
& \|u\| \rightarrow \infty
\end{aligned}
$$

where $C>0, M C>0$.

\section{REFERENCES}

[1] S. J. Li and M. Willem, "Applications of Local Linking to Critical Point Theory," Journal of Mathematical Analysis and Applications, Vol. 189, No. 1, 1995, pp. 6-32. doi:10.1006/jmaa.1995.1002

[2] X.-L. Fan and Y.-Z. Zhao, "Linking and Multiplicity Results for the p-Laplacian on Unbounded Cylinders," Journal of Mathematical Analysis and Applications, Vol. 260, No. 2, 2001, pp. 479-489. doi:10.1006/jmaa.2000.7468

[3] Q. S. Jiu and J. B. Su, "Existence and Multiplicity Results for Dirichlet Problems with p-Laplacian," Journal of Mathematical Analysis and Applications, Vol. 281, No. 2, 2003, pp. 587-601. doi:10.1016/S0022-247X(03)00165-3

[4] P. H. Rabinowitz, "Periodic Solutions of Hamiltonian Systems," Communications on Pure and Applied Mathematics, Vol. 31, No. 2, 1978, pp. 157-184. doi: $10.1002 / \mathrm{cpa} .3160310203$

[5] Q. Jiang and C. L. Tang, "Existence of a Nontrivial Solution for a Class of Superquadratic Elliptic Problems," Nonlinear Analysis, Vol. 69, No. 2, 2008, pp. 523-529. doi:10.1016/j.na.2007.05.038

[6] S. X. Luan and A. M. Mao, "Periodic Solutions for a Class of Non-Autonomous Hamiltonian Systems," Nonlinear Analysis, Vol. 61, No. 8, 2005, pp. 1413-1426. doi:10.1016/j.na.2005.01.108 